\title{
Improvement of Smart Wearable Device to Assure Right Direction for Elderly People
}

\author{
Nimai Chandra Das \\ Department of Electrical and \\ Electronics Engineering \\ Daffodil International University
}

\author{
Rony Shaha \\ Department of Electrical and \\ Electronics Engineering \\ Daffodil International University
}

\author{
Md. Elias Hossain \\ Department of Software \\ Engineering \\ Daffodil International University
}

\author{
Md. Sazzad Sarker \\ Department of Electrical and Electronics \\ Engineering \\ Daffodil International University
}

\author{
Md. Wahidur Rahman \\ Department of Computer Science and Engineering \\ Mawlana Bhashani Science and Technology \\ University
}

\begin{abstract}
The aim of this study is to create an efficient monitoring system for elderly people in society. As the number of elderly people is increasing, most of the aged people rest their lives in the old-age home. During leading their life in the old-age home, aged people face many problems who reach 58-62 or above of their age. This problem can be listed such as falling sudden illness, incapability of hearing clearly at a certain normal decibel level, unable to move anywhere without the co-operation of others. In this, we have introduced an effective solution that has ability to monitor older persons, a low-cost alarming system and so on. Those can be ameliorated to some extent. Recently, advance wearable and sensor technologies have improved the prospects of this service for caring for aged people. In this proposed project can ensure that the care of aged people by smart device services which can give voice direction to go to the desired room between many rooms and take immediate help if it's necessary though pressing a single button with the alarm system.
\end{abstract}

\section{Keywords}

Voice direction, Emergency system, Micro-controller, Smart wearable device, Aged people, Master device, Slave device.

\section{INTRODUCTION}

As we all know, that population leads us to some significant challenges to our societies. Moreover, the increasing rate of elderly populations is promising more health care services, including those of old-age homes, hospitals, and nursing homes as well as our houses. Elderly people are unable to take care of themselves, it is very critical for a person who takes care of aging people in an old-age home. A prospective solution that can improve the care of aged people in old-age houses with the help of wearable technologies. Fortunately, the advent and advance of wearable solutions have provided a way to develop feasible devices for elderly care.

In recent years, smart-watch, smart mobile phones, and smart clothing are the elementary products of embedded wearable technologies with necessary caring functions. All of them have attractive advantages for delivering health information. Quite a lot number of smart wearable devices have been designed for elderly care. Some of those can recognize the physical activity and monitoring specific sections however some of those smart wearable devices cannot provide support actively or precisely.
This research proposed a design for those who are living their rest of life in an old-age home. As the aim of this paper is to provide support to aged people through the use of wearable solutions for their care. We have identified wearable technologies that can meet the demands or can be improved to satisfy the needs of elderly care issues. The challenge with smart-care technologies is to support an old person in the oldage home environment that is safe and secure in order to reduce falls, disability, stress, fear or social isolation Contemporary smart-care technology systems are multipurpose in functionality and user-friendly. This project usually aims to perform functions without disturbing the user and without causing any pain, inconvenience or movement restrictions. Through this system, the users are able to take immediate help while pressing a single button. When the user presses the button, a connection with another room a voice will turn on and the user can listen that will enable enrich user to take decisions in which room he/she has to go. The function is to build for the consideration of age people as we know they are not capable of remembering everything correctly at the time when they go around the old-age home environment.

\section{RELATED WORK}

In, 10 Feb 2017, Zhihua wang, Zhoachu yang and Tao dong [1]. They have worked a project which is wearable technologies for elderly care that can accurately track indoor position, recognize physical activities and monitor vital signs in real-time by using many sensors and GPS, wifi communication.

In October 2015, Alvin Jacob, Wan Nurshazwani Wan Zakaria and Mohd Razali Bin Md Tomari [2] have worked with the master and slave method of Bluetooth communication at flex sensor with Bluetooth module that is the implementation of Bluetooth communication in developing a mobile measuring device to measure human finger movement.

In March 2017, Ankit Panvalkar, Shubham Sawant, Varun Gokhale, Rushabh Shah, Dr. T. Rajani Mangala [3]. They work to configure master and slave communication through their paper,master-slave configuration base Bluetooth controlled robots.

Some comparable work has been discovered for elderly people that can precisely follow indoor position, perceive physical exercises and watch fundamental signs continuously by utilizing numerous sensor, gaps and Wi-Fi correspondence 
(Wang, Z., Yang, Z., \& Dong, T.2017)

Some research papers focus around assessing and testing the use of Bluetooth correspondence in a versatile estimating gadget (Jacob, A., Zakaria, W. N. W., \& Tomari, \& M. R. B. M., 2015)

Some comparable paper proposes an Intelligent Home Navigation System (IHNS) which contains a wheelchair, voice module, and route module. It tends to be utilized by an old or physically provoked individual to move inside the home with no trouble (Megalingam, R. K., Nair, R. N., \& Prakhya, S. M. 2011)

Related work Ambient Assisted Living (AAL) framework has been structured so as to make better living conditions for more established individuals. Along these lines, individuals can live autonomously longer in their own home with improved personal satisfaction (Mainetti, L., Patrono, L., Secco, A., \& Sergi, I, 2016)

Some work introduces an IoT-prepared answer for the older living help which can screen and enlist patient's indispensable data just as to give systems to trigger alerts in crisis circumstances. (Pinto, S., Cabral, J., \& Gomes, T, 2017)

Some related research demonstrates a user assessment assessing the receipt and fear of this new technology (Portet, F., Vacher, M., Golanski, C., Roux, C., \& Meillon, B, 2013)

Similar work proposes to develop a Wireless Sensor Network based smart home system for such elder people to help them ease their labor and deliver them harmless, sound and safe living (Ransing, R. S., \& Rajput, M, 2015).

\section{PROPOSED MODEL AND METHODOLOGY}

In this proposed solution, we enlighten our system with one master device and many slave devices. This slave device will be connected when the desired button is pressed. Figure 1 shows the proposed model of our system. Here, there are three slave devices and also four buttons connected to the master device including emergency help button. The slave devices will connect automatically while pressing the button which will guide the user to go towards the right path within a selected area where the user wants to go. When system finds a button is pressed a predefined slave device will be connected and produce voice output through the speaker. If the user presses wrong button instead of right, he/she will not able to go to respected places. Users can overcome this situation by pressing help button.

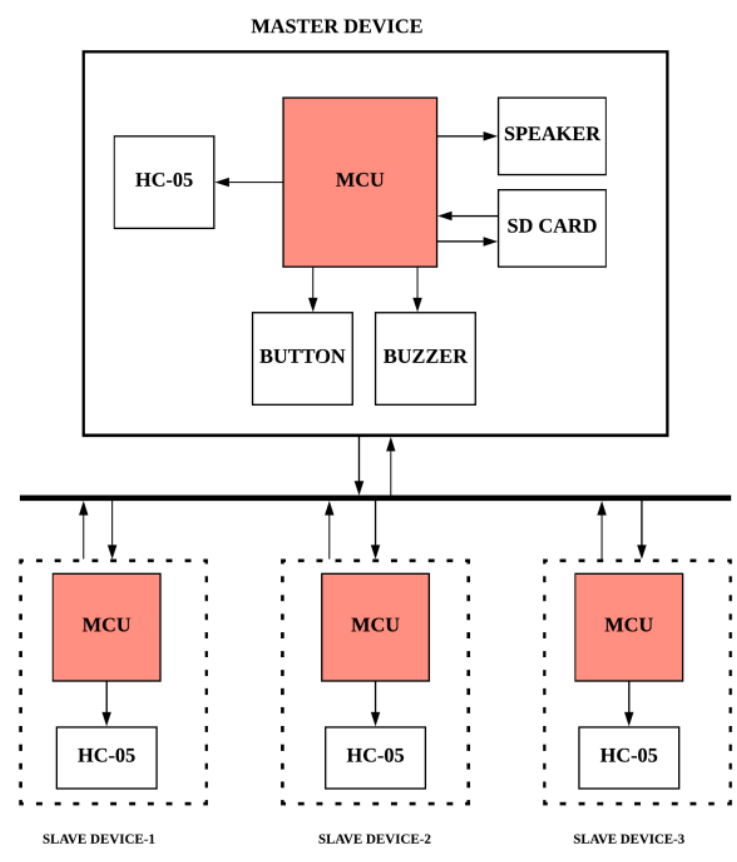

Fig 1: Working flowchart of the proposed solution

Wearable technologies are basically used for old-age people is to care for them in the indoor position and make their safety from certain injuries and illnesses. This technology also assures proper direction for those aged people through this project. In this project, we try to develop a smart care device by using a master and slave method of Bluetooth communication.

The communication of this proposed solution is SPI with Bluetooth communication which can operate automatically in synchronous mode between two or more devices strongly with proposed smart care wearable devices in the old-age home environment. The type of master-slave controls a method which has the merits of both unilateral and bilateral ones, is proposed. A master-slave controls the system and the method of operation wherein the master elements have substantial able absolute invasive control over the functions and capabilities of slave devices. These slave devices are linked within the networks and wherein the master device can exercise latent control over the slave devices. Moreover, one device has a unidirectional control with two or more devices in our proposed system. We enriched our system with one master device and two slave devices using Bluetooth communication. In our proposed system, voice recorded files are executed in the form of modulation defined through master device. The file format of the recorder clips is ".wav". Block Diagram of the project is given in Figure 2 and 3. In Figure 2 we have placed micro-controller to control overall project. Here MCU is enriched with buzzer, speaker and Bluetooth module. All those parts are discussed earlier in this section. Again, slaves will be consist of only external power source and Bluetooth module. 


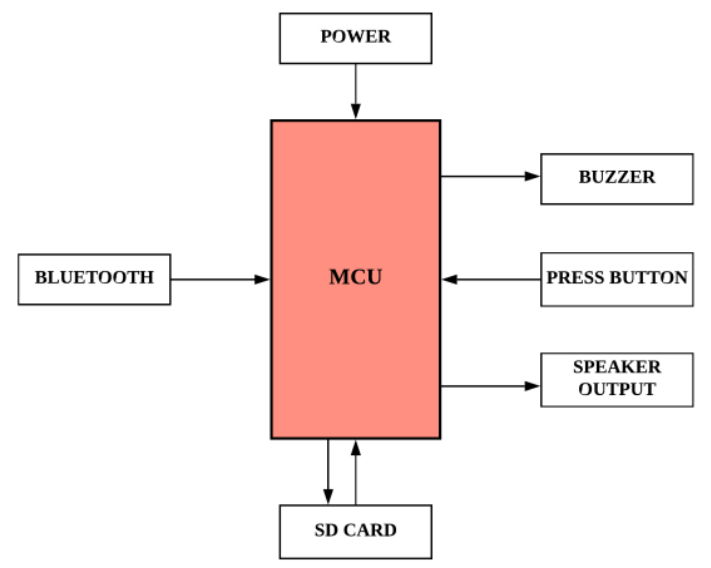

Fig 2: Working flowchart of the proposed master circuit

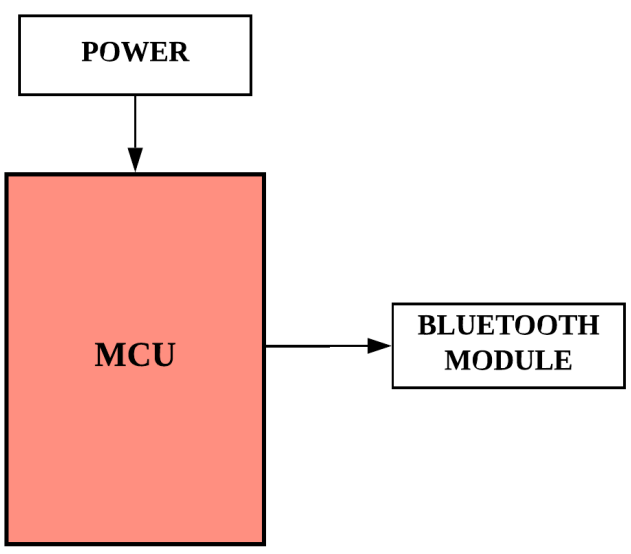

Fig 3: Working flowchart of the proposed slaves

\section{PROJECT IMPLEMENTATION}

\subsection{A Master Device}

In the master device, the power supply is provided to the Arduino, Bluetooth module, speaker, and Arduino SD card reader. In the master device, Bluetooth is used to ensure communication between the master device and a slave device. When the master device is powered up, it effectively searches slaves in order to connect with corresponding slave devices. Bluetooth requires a pairing state. Hence, it controls the slave. While pressing the button on the master device, one of the desirable slave devices starts to communicate among them. After successfully communication is built in the slave devices, the command is then executing in the master device.

\subsection{Slaves Devices}

These devices are also powered up individually through the Arduino board. These slave devices find a power source, they are ready to communicate with the master device. These devices are waiting for a signal from the master device. When the Bluetooth connection is established, it will able to return the same data to the master device and the voice command file will be executed. This process effectively reduces overall circuit complexity without making any changes in the master device.

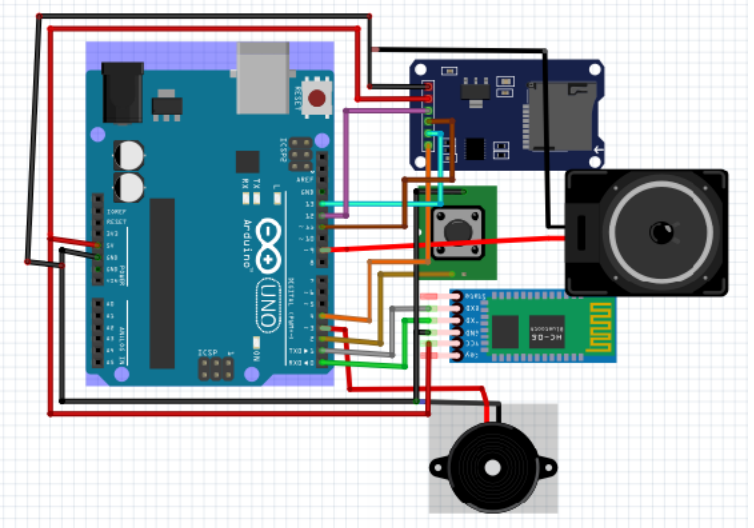

Fig 4: Circuit Diagram of Master Device

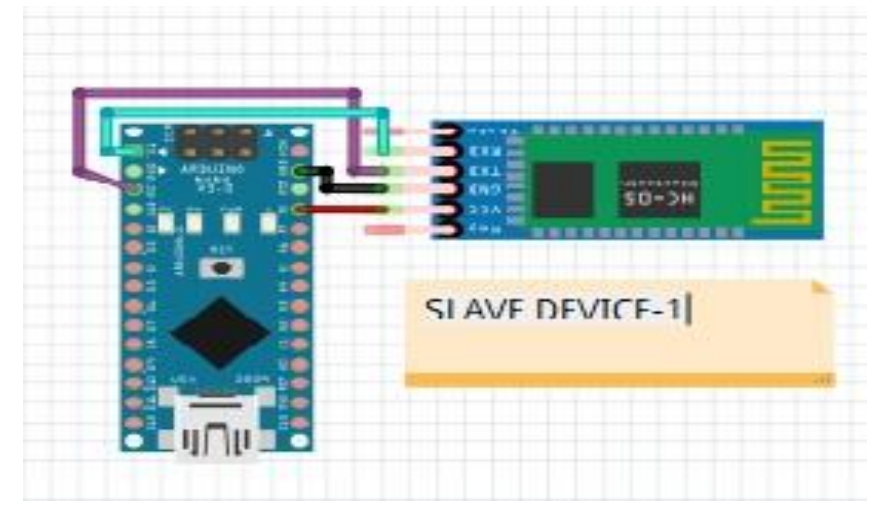

Fig 5: Circuit Diagram of Slave Devices

The above Figures 4 and 5 show the overall hardware configuration based on the hardware development which is accomplished by ourselves to test and experiment within the proposed solution. The design has been adapted into some experiments to test the capability and usability in the real world. Bluetooth module ensures wireless communication which essentially allows the connected devices to send and receive data between master and slave devices. The Bluetooth module used in this testing is HC-05 which is capable of acting as both master and slave devices. As far as we know that, the Bluetooth module HC-05 v2.0 model uses Gaussian Frequency Shift Keying modulation, the maximum transfer rate is $2.1 \mathrm{Mbps}$ and operating transmission distance varied in a range between 20 to $30 \mathrm{~m}$ in the free space. On the other hand, the Baud rate is also an important factor that is needed to consider data transmission. Baud rate is needed to set up $1200 \mathrm{bps}$ to $1382400 \mathrm{bps}$. But $9600 \mathrm{bps}$ is used for all the preliminary tastings. The data flow of the hardware sections is shown in Figure 6.

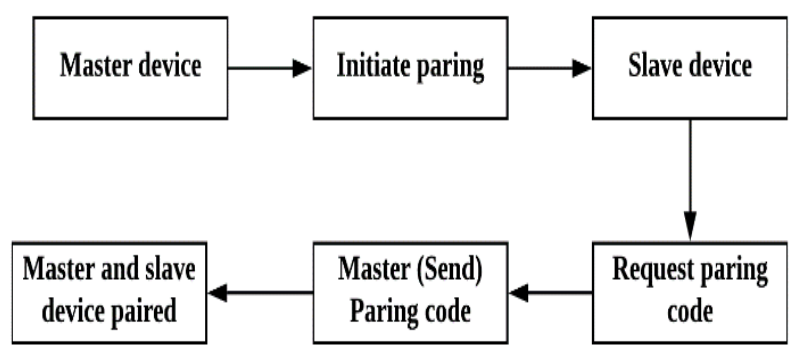

Fig 6: Flow Diagram of Master-Slave Communication using Bluetooth pairing 


\section{RESULT AND DISCUSSION}

In this section, we are going to discuss the results and discussion. After successfully making our prototype, we have performed several types of testing. We have found Bluetooth device has an accuracy rate of $100 \%$ and data passing rate is almost $90 \%$. The error rate of communication with master and slave is less than $5 \%$. The use of buttons to connect with the slave device and get help right away. As soon the user presses a button to connect to a pleasant device system that will connect the right way less than 4 seconds latency. As the connection to the device will be established, the user gets a vocal direction what will be the user's next step/move.

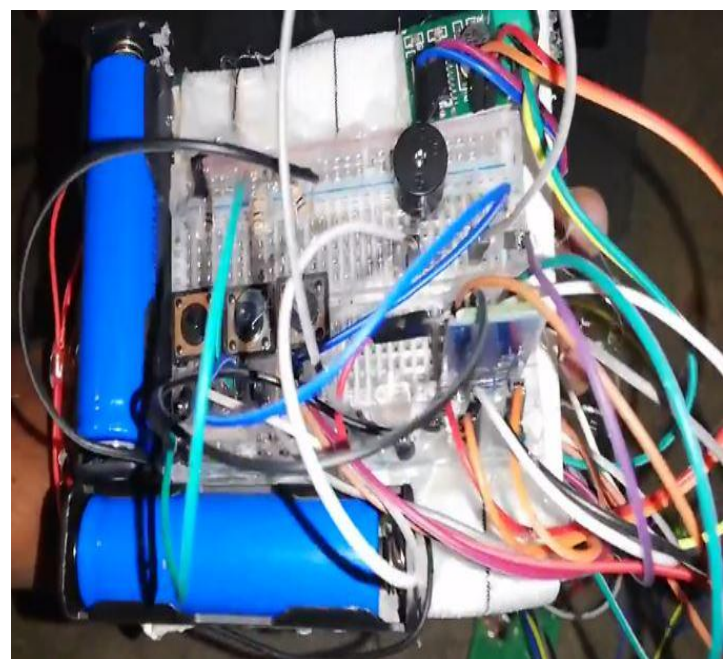

Fig 7: Hardware setup for mater device

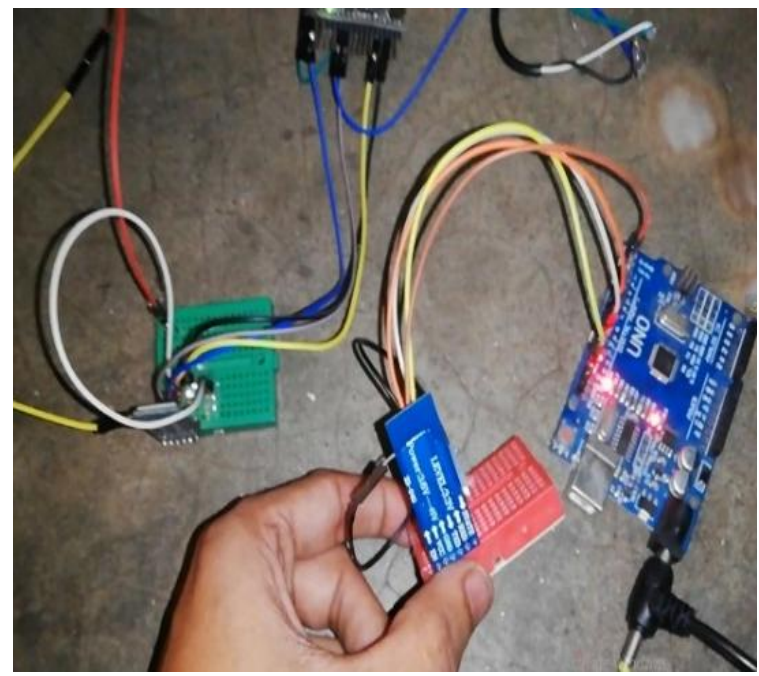

Fig 8: Hardware setup for the slave device

By doing the same process user communicates with all the devices around the local environment. Due to the use of efficient programming logic system works fluently. Within the communicating range of 10-14 meter systems output is faster. Here microcontroller takes approximately 10 seconds to switch connections between devices.

\section{Experimental Data}

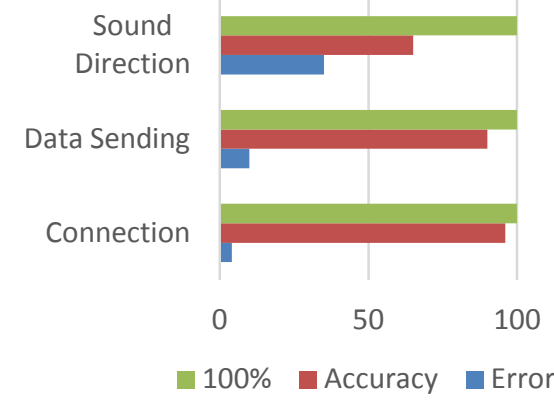

Fig 9: Chart of Experimental Data Acquisition

Figure 7 and 8 shows the hardware configuration of our developed project. Figure 9 shows experimented data analysis using Bar chart. Basically, in this chart we have tried to figure out respective experiments such as sound direction, data sending time as well as connection establishment time. We have also provided accuracy and error percentage.

\section{FUTURE SCOPE AND CONCLUSION}

Wearable technologies for old people can be very important as they are going to have an assistant by the side for help. This system can be helpful as old people tend to forget very easily. Japan is facing an aging problem as most of the population is over 65 years old. This situation requires a lot of care which is hard to get these days anywhere around the world. If we try and shrink that problem with modern technologies we might have a solution with proper care. Few push-buttons are required to remember as this is very important for helping and direction to anything. This proposed system also might help as a medication reminder as well. A complete set of assistance for our aging population. We have used the technologies which can be viable for a short period of time. As technologies changing every single day, we have to look forward to increasing the helping capacity. Currently, we are working on a local system. But in the future we will include heart rate monitoring system and sleep tracking system and direct contact with a doctor. A conversational Assistant for aging people will also be added when they don't have any relatives to talk with them. Furthermore, we will work on the learning curve for prediction of the next possible steps of movement. Again, Home automation capabilities and pathfinder between indoor and outdoor areas also can be possible to design a compact product for market availability.

\section{REFERENCES}

[1] Zhihua wang, Zhoachu yang and Tao dong. "A review of wearable technologies for elderly care that can accurately track indoor position recognize physical activities and monitor vital signs in real time",10 February 2017.

[2] Alvin Jacob, Wan Nurshazwani Wan Zakaria and Mohd Razali Bin Md Tomari.: implementation of Bluetooth communication in developing a mobile measuring device to measure human finger movement.ARPN Journal of engineering and applied Sciences, vol.10,No 19,October 2015.

[3] Ankit Panvalkar, Shubham Sawant, Varun Gokhale, Rushabh Shah, and Dr. T. Rajani Mangala.: Master-slave configuration based Bluetooth controlled robots. International Journal of Advanced Research in Electrical, 
Electronics and Instrumentation Engineering, vol.6, Issue 3, March 2017.

[4] Wang, Z., Yang, Z., \& Dong, T. (2017). A review of wearable technologies for elderly care that can accurately track indoor position, recognize physical activities and monitor vital signs in real time. Sensors, 17(2), 341.

[5] Jacob, A., Zakaria, W. N. W., \& Tomari, M. R. B. M. (2015). Implementation of Bluetooth Communication in Developing a Mobile Measuring Device To Measure Human Finger Movement. ARPN Journal of Engineering and Applied Sciences, 10(19), 8520-8524.

[6] Megalingam, R. K., Nair, R. N., \& Prakhya, S. M. (2011, February). Automated voice based home navigation system for the elderly and the physically challenged. In 13th International Conference on Advanced Communication Technology (ICACT2011) (pp. 603608). IEEE.

[7] Mainetti, L., Patrono, L., Secco, A., \& Sergi, I. (2016,
July). An IoT-aware AAL system for elderly people. In 2016 International Multidisciplinary Conference on Computer and Energy Science (SpliTech) (pp. 1-6). IEEE.

[8] YPinto, S., Cabral, J., \& Gomes, T. (2017, March). Wecare: An IoT-based health care system for elderly people.In 2017 IEEE International Conference on Industrial Technology (ICIT) (pp. 1378-1383). IEEE

[9] Portet, F., Vacher, M., Golanski, C., Roux, C., \& Meillon, B. (2013). Design and evaluation of a smart home voice interface for the elderly: acceptability and objection aspects. Personal and Ubiquitous Computing, $17(1), 127-144$

[10] Ransing, R. S., \& Rajput, M. (2015, January). Smart home for elderly care, based on Wireless Sensor Network. In 2015 International Conference on Nascent Technologies in the Engineering Field (ICNTE) (pp. 15). IEEE. 